\title{
COVID-19 Contact Tracing using Blockchain
}

This paper was downloaded from TechRxiv (https://www.techrxiv.org).

\section{LICENSE}

CC BY 4.0

SUBMISSION DATE / POSTED DATE

$18-02-2021$ / 25-02-2021

\section{CITATION}

Hasan, Haya R.; Salah, Khaled; Jayaraman, Raja; Yaqoob, Ibrar; Omar, Mohammed; Ellahham, Samer (2021): COVID-19 Contact Tracing using Blockchain. TechRxiv. Preprint.

https://doi.org/10.36227/techrxiv.14052320.v1

DOI

10.36227/techrxiv.14052320.v1 


\title{
COVID-19 Contact Tracing using Blockchain
}

\author{
Haya R. Hasan, Khaled Salah, Raja Jayaraman, Ibrar Yaqoob, Mohammed Omar, Samer Ellahham
}

\begin{abstract}
Contact tracing has widely been adopted to control the spread of Coronavirus-2019 (COVID-19). It enables to identify, assess, and manage people who have been exposed to COVID19 , thereby preventing from its further transmission. Today's most of the contact tracing approaches, tools, and solutions fall short in providing decentralized, transparent, traceable, immutable, auditable, secure, and trustworthy features. In this paper, we propose a decentralized blockchain-based COVID-19 contact tracing solution. Contact tracing can greatly suffice the need for a speedy response to a pandemic. We leverage the immutable and tamper-proof features of blockchain to enforce trust, accountability, and transparency. Trusted and registered oracles are used to bridge the gap between on-chain and off-chain data. With no third parties involved or centralized servers, the users' medical information is not prone to invasion, hacking, or abuse. Each user is registered using their digital medical passports. To respect the privacy of the users, their locations are updated with a time delay of 20 minutes. Using Ethereum smart contracts, transactions are executed on-chain with emitted events and immutable logs. We present details of the implemented algorithms and their testing analysis. We evaluate the proposed approach using security, cost, and privacy parameters to show its effectiveness. The smart contracts code is publicly made available on GitHub.
\end{abstract}

Index Terms-COVID-19; Blockchain; Ethereum Smart Contracts; Transparency; Security Analysis; Contact Tracing

\section{INTRODUCTION}

The year 2020 has witnessed the spread of Coronavirus2019 (COVID-19) all over the world. The major surge of the virus globally shook the foundations of the health sector which weren't adequately prepared and couldn't respond with high efficiency. Hence, the advancements in technology can help in restoring human lives across the world. In efforts to mitigate the unprecedented spread of COVID-19, contact tracing applications have widely been developed [1]-[3]. Contact tracing applications are believed to be able to break the chain of COVID-19 infections [4]. Contact tracing or proximity tracing can be used to identify how close someone has been to other contacts regardless of the context and one of those people in contact is positive to the pandemic virus. Tracing back all the possible contacts to the positive case and informing them about the possibility of being infected is part of contact tracing. This is essentially important to stop the further spreading of COVID-19.

Haya R. Hasan, Khaled Salah, and Ibrar Yaqoob are with the Department of Electrical Engineering and Computer Science, Khalifa University of Science and Technology, Abu Dhabi 127788, United Arab Emirates.

Raja Jayaraman and Mohammed Omar are with the Department of Industrial and Systems Engineering, Khalifa University of Science and Technology, Abu Dhabi 127788, United Arab Emirates.

Samer Ellahham is with Heart \& Vascular Institute, Cleveland Clinic Abu Dhabi, Abu Dhabi, United Arab Emirates.

Corresponding author: ibraryaqoob@ieee.org
The deficits of the current contact tracing technologies and solutions should be overcome to make contact tracing more successful. Three main issues; namely, privacy, accountability, and transparency must be considered when developing contact tracing applications [5]. Privacy of the users should be maintained where the data of users and their personal information should not be stored on centralized servers that are prone to hacking and abuse. On the other hand, accountability refers to the role of the technology held accountable for the decisions taken based on the lack of precision in proximity measurements. Additionally, transparency is vital when trying to communicate with the individuals of the societies. Application users should know how their input is being used, how it is processed, and where is the output used and distributed. However, the current systems do not consider the privacy of the users and are not transparent enough. Hence, users are discouraged from using the contact tracing applications [6].

In this paper, we aim to curb the spread of COVID-19 infections through a blockchain-based contact tracing solution. We leverage the use of the intrinsic features of blockchain technology to deal with the contact tracing challenges. Therefore, the implemented solution respects the privacy of its users. It is immutable, transparent, and accountability is a built-in feature by design. Blockchain is a distributed shared ledger that is decentralized with tamper-proof and immutable logs [7]. It is a linked list where all nodes keep a local copy of all the nodes [8]. Blockchain has a wide range of applications from e-commerce, to supply chain management [9]. In the context of the COVID-19 pandemic, blockchain has proven to be a versatile technology that can be used in several applications to mitigate the spread of infections [10], [11].

Contact tracing is one of the many ways that blockchain has proved to be useful to eradicate the effects of the pandemic. Using Ethereum blockchain with the added programmable logic using smart contracts allows the different participants to be transparent, accountable, and trusted. Our solution eliminates third-party servers, centralization, and identity abuse. It relies on the distributed ledger's immutable logs to enforce transparency and trust. All transactions taking place on-chain are signed by their creator. Hence, every on-chain participant is held accountable for their action.

\section{A. Related Work and Contributions}

Herein, we review the existing literature available on contact tracing applications and their integration with blockchain.

The authors in [12] presented a survey on contact tracing applications used for COVID-19. The authors were aimed to highlight the key differences and features of the different applications especially in certain attributes related to the system architecture and design, data security and privacy as well 
as attacks and vulnerabilities. The authors outlined different applications such as Trace Together, Covid Safe, Covid Watch, and EpiOne. The authors concluded that each application based on its architecture whether centralized, decentralized, or hybrid has its pros and cons. They also emphasized on the adoption rate between the users and how it can increase with transparency. Decentralization in their context referred to reducing the load on the servers and increasing it on the user devices. They did not tackle the aspect of decentralization using blockchain-based solutions.

The authors in [11], [13], [14] discussed that blockchain can be used to create a decentralized contact tracing solution. Its intrinsic features help in ensuring transparency, trust, pseudoanonymity, and decentralization. In [15], the authors presented a blockchain-based contact tracing framework. They highlighted the privacy concerns in the available contact tracing applications due to the use of centralized servers and suggested a blockchain-based design that could overcome the privacy issues of the existing solutions. Their solution does not show any implementation details or testing results. On the other hand, BeepTrace [16] emphasized on the importance of using blockchain in contact tracing to add trust, transparency, and privacy. Hence, in their solution, they introduce two types of blockchain networks one for the tracing and the other one for the notifications. BeepTrace shows promising results when compared to other solutions presented by the authors in terms of cost, security, and privacy. However, its solution depends on using trusted third parties such as the 'Geodata solvers' servers as well as a Public Key Infrastructure (PKI).

To overcome the use of third parties, the authors in [17] proposed a blockchain network named Bychain. In the network, they amend the fields of the used blockchain blocks to suit their block proposal and validation scheme. Their solution also depends on short-range communication (SRC). The testing is done on the implemented blockchain network design to test the messages' latency, power consumption, computation, and storage limitations. Their main objective is to test the newly created Bychain which offers limited information on results related to COVID-19 contact tracing. Moreover, the study conducted in [18] made use of an Internet of Things (IoT) hardware model that uses passive RFID transceivers. The solution ensures that users remain anonymous until a user tests positive for the COVID-19 virus. The study presents smart contract (SC) codes, as well as hardware details. However, the study does not present a complete architecture or design of the proposed blockchain solution. Furthermore, the study conducted in [19] presents a contact information sharing and risk notification system using blockchain. Their solution uses Bluetooth as a short-range communication technology. Their solution concentrates on quantifying the possibility of a user infection based on the information provided by the user or from the shared information. The status of the user changes based on information entered by the users. The possibility of infection for other contacts is calculated using an equation proposed by the authors.

Numerous research efforts have been conducted on COVID19 contact tracing [12]. However, most of the existing solutions are based on centralized servers or third parties [20]. Fig- ure 1 presents a basic architecture of the centralized solution that depends on servers. The application users would send their location information to centralized data centers. On the other hand, the COVID-19 testing centers send the COVID-19 test results to the centralized servers. The server would do all the processing based on the input received by the application users and the testing centers. The result containing the COVID-19 contacts list is then sent to the COVID-19 testing centers to take the needed action. Hence, in such an architecture, the users' information is all stored in the servers. Also, there is no transparency, and everyone involved needs to trust the server and each other in being honest. Furthermore, such a system is prone to being a single point of failure. Unlike the aforementioned solutions, our solution leverages blockchain technology and utilizes its built-in security features to present transparent, tamper-proof, and immutable transactions between all participating entities.

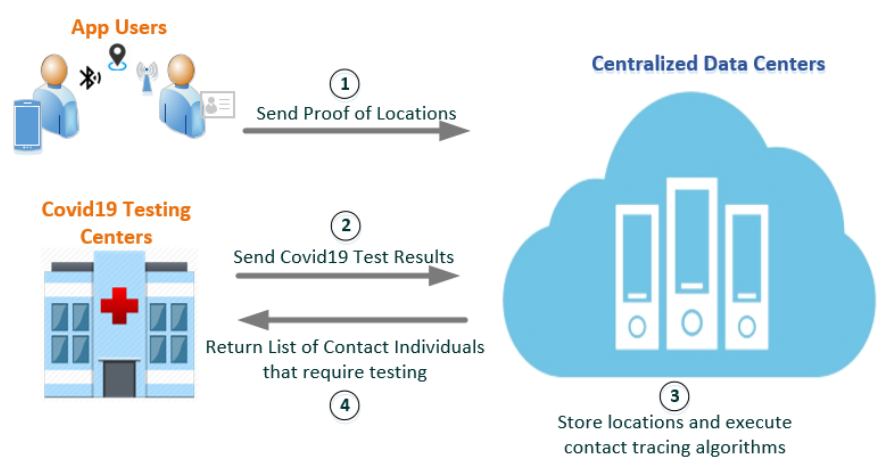

Figure 1: Centralized contact tracing applications using servers

The main contributions of this paper can be summarized as follows:

- We showcase a blockchain-based approach to enable and provide COVID-19 contact tracing in a manner that is fully decentralized, transparent, traceable, immutable, auditable, secure, and trustworthy.

- We integrate the Ethereum blockchain with on-chain registered oracles to execute the contact tracing algorithms to put down the extra burden and save cost.

- We develop smart contracts along with algorithms to implement functionalities and define rules regarding COVID-19 contact tracing applications. The smart contracts code is publicly made available on GitHub ${ }^{1}$.

- We perform the cost analysis to show feasibility and affordability of the solution, and present security analysis to show that our smart contracts are secure enough against well-known vulnerabilities and attacks.

- Our proposed blockchain-based COVID-19 contact tracing solution is generic and can be easily customized as per the needs and requirements of various types of contact tracing applications focusing on other diseases.

The rest of the paper is organized as follows. Section II presents the design details of the proposed blockchain-based solution followed by the implementation details in section III including the smart contracts and algorithms. Section IV

\footnotetext{
${ }^{1}$ https://github.com/smartcontract694/covid19/tree/master
} 


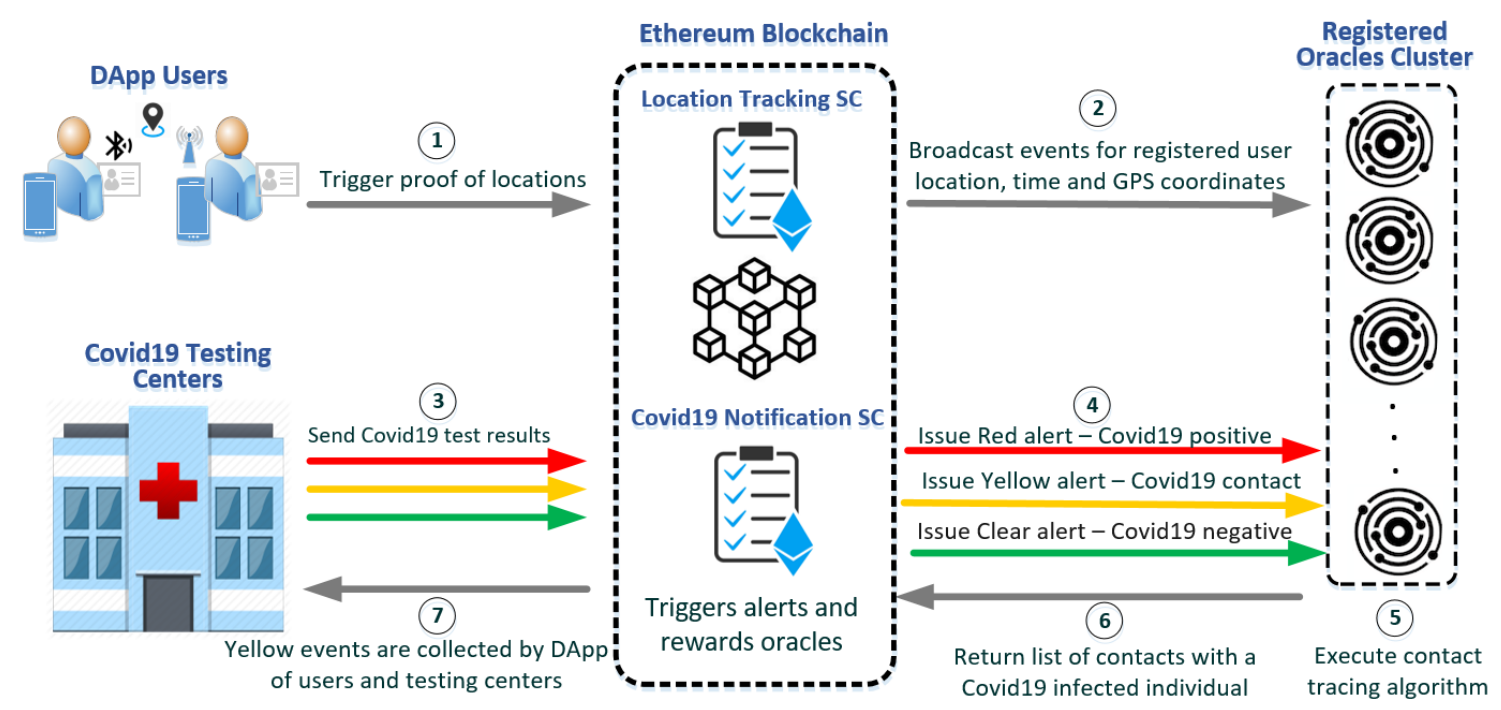

Figure 2: System diagram of the proposed blockchain-based COVID-19 contact tracing solution

presents the testing details of the proposed system followed by section $\mathrm{V}$ which showcases the security and cost analysis along with discussing the privacy and generalization aspects. Section VI concludes the paper.

\section{SySTEM DESIGN}

This section presents the system design of our proposed blockchain-based COVID-19 contact tracing solution. Figure 2 shows the system components of the solution along with the interacting entities. The decentralized application (DApp) users employ their smartphones to trigger proof of locations that are logged on-chain through the smart contracts. Consequently, the registered oracles use the broadcast events and locations in their contact tracing algorithms. On the other hand, the COVID-19 testing centers send COVID-19 results to the blockchain to trigger alerts as needed. The alerts issued vary depending on the received result. Those emitted events are alerts used to notify the registered oracles to execute the contact tracing algorithm. The registered oracles return the list of contacts of the possibly infected individuals to the smart contract.

\section{A. DApp Users}

DApp users employ the downloaded contact tracing application to trigger proof of locations. Those proof of locations have the latitude, longitude, time, and date that are communicated to the 'Location Tracking SC'. Communication happens when a user is within contact with another user at a distance of fewer than 2 meters only. This allows saving on power and cost. Smartphones find each other through short-range communication such as Bluetooth. Other means are also possible using WiFi or mobile data. Each user's proof of location and contact is stored on the ledger for an immutable log that is trusted when needed. Furthermore, to preserve the privacy of the user there is a 20-minute delay before sending proof of location. This is done to ensure that the current location of the user is not known. Hence, the user's privacy is not abused through invasion.

The application users must be registered to use the contact tracing application and on-chain functions. The registration is done through the affiliated testing centers and every Ethereum address (EA) is associated with bio-metrics data. Furthermore, the registered users have a digital medical passport that has all their medical information [21]. Health Passport is identified as an emerging technology in Gartner's 2020 Hype Cycle [22] and this research uses the concept of the medical passport from our previous work conducted in [21] as part of the solution. The users' right to convey the information included in their medical passports to other entities is based upon their choice [21]. All the information on-chain is stored using IPFS hashes. The information is kept confidential until the user allows a certain entity to get access to the information. Hence, only then this information is disclosed to the entity authorized by the user [21]. Therefore, authorization is needed by the users to disclose any of their information.

\section{B. COVID-19 Testing Centers}

COVID-19 testing centers are specialized in conducting COVID-19 tests. They would communicate the result with the 'COVID-19 Notification SC' to issue an alert based on the result. The COVID-19 testing centers also await a response from the COVID-19 Notification SC. This response helps them in finding out the people of contact that need to be quarantined, tested, and treated if needed. The SC in response issues yellow alerts that are based on the result of the contact tracing algorithms run by the registered oracles. Hence, the COVID-19 testing centers are the point of contact between the test takers and the COVID-19 Notification SC.

\section{On-chain Smart Contracts}

Using the Ethereum blockchain, it is possible to execute smart contracts that carry programmable logic. Digital assets 


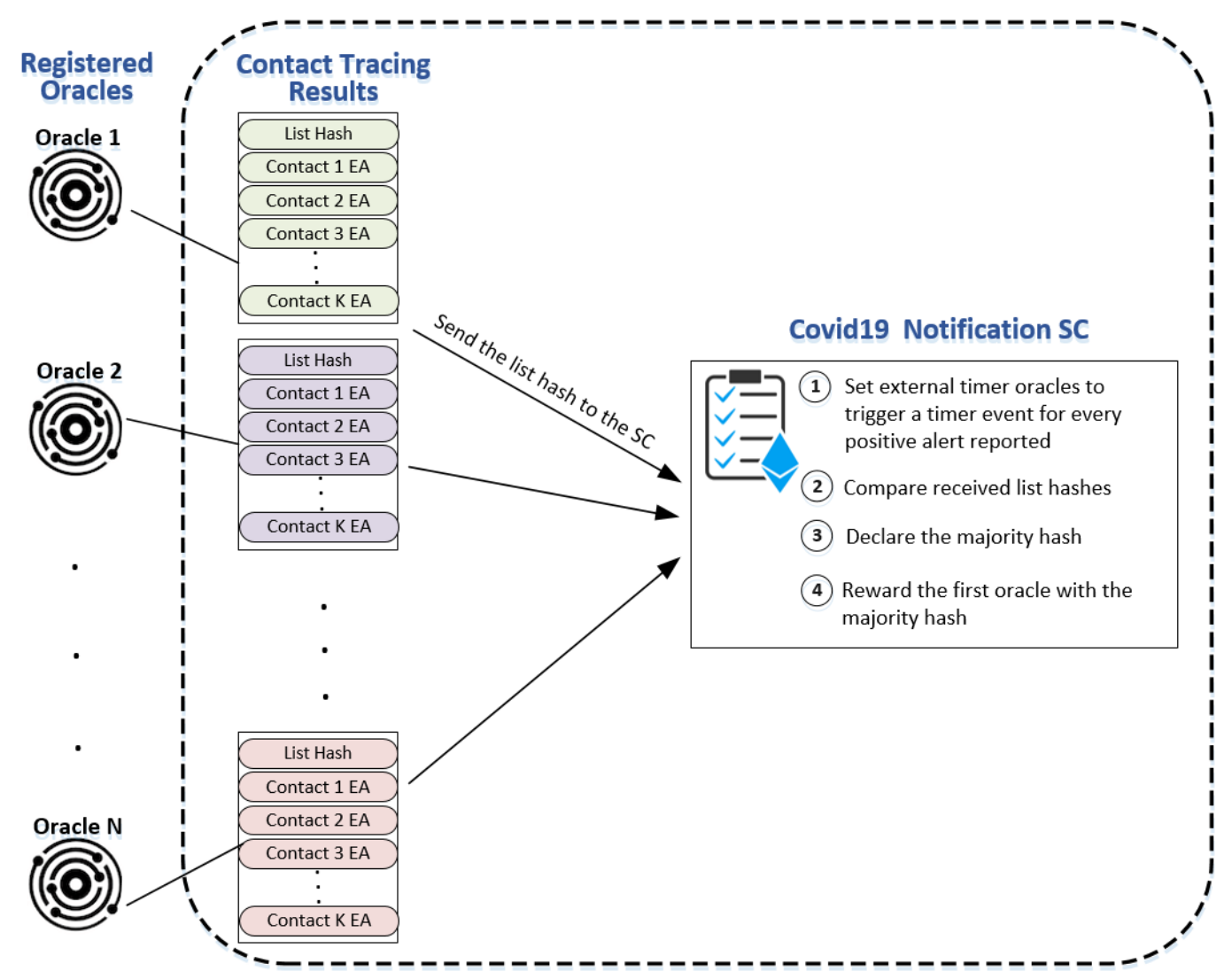

Figure 3: Oracles communication with the COVID-19 Notification SC

as well as 'Ether' can be controlled using the logic presented in the SC. Events are emitted in logs to ensure that the actions taken are transparent and immutable. This establishes trust among all the participating entities on-chain. In our proposed solution, we have implemented in our design two smart contracts.

1) Location Tracking SC: In the location tracking SC, locations of the DApp users are logged. This is the main task of the smart contract. This is a very vital step to ensure that all contacted individuals can be traced based on their locations. The decentralized applications communicate to the blockchain the locations of their users through the location tracking smart contract. The longitude, latitude, as well as time, are all sent to be immutably logged. The smart contract, on the other hand, can emit events that get broadcast to all the registered oracles as well as participating entities. This ensures that the GPS coordinates are available when needed for contact tracing.

2) COVID-19 Notification SC: The COVID-19 notification smart contract handles COVID-19 alerts. The alerts are of three types based on the COVID-19 test results. Red, yellow, and green are the three colors used to identify the alert types. The different alerts are issued by the smart contract after the COVID-19 test results are sent by the COVID-19 testing centers. The issued alerts are then used to update the on-chain profiles of the users as well as alert the registered oracles of the new updates. The registered oracles use the logged COVID-19 alerts on-chain to know whether a contact list is required. A red or yellow alert based on the testing center results indicates that the contact tracing algorithm must be executed. However, it does not require to be executed in the case of a green alert. The registered oracles execute the contact tracing algorithm and return the list of contacts that could be infected. The individuals' EAs are entered into the smart contract and the results are emitted as yellow alerts. Those alerts are used by the testing centers as well as DApp users to notify the users that they could be infected by the virus. The status of the users would turn to yellow based on the contact tracing information.

\section{Registered Oracles}

Oracles are used to connect the blockchain to the off-chain data and input. To maintain the deterministic nature of the decentralized blockchain, no external calls to any APIs are allowed. Therefore, oracles are the bridges used to connect the blockchain to the outside world; hence, named as blockchain middleware [23].

1) The Oracle Problem: The oracle problem is one of the common problems in the Oracle-based systems [24]. Choosing only one oracle as the main source of information from the outside world leads to create dependency on a single entity that can result in a single point of failure [25]. Furthermore, it is hard to assume that a single oracle can be trustworthy given the possibility that it can be hacked and depreciated. To solve the oracle problem, a network of oracles need to be used [26]. Hence, we used a cluster of registered oracles 
that communicate with the Ethereum smart contracts on-chain. This ensures that the blockchain has access to reliable secure off-chain data. Decentralized networks of oracles are a solution that maintains the distributed nature of blockchain at the same time along with ensuring that the blockchain can have access to real-world data and information.

2) Oracles Registration: Oracles need to register so that they become authorized to access the functions in the smart contracts. In our design, the 'Oracles SC' is the smart contract responsible for the services of the oracle such as registration, awarding, and penalizing. The 'Oracles SC' owner is an authorized entity that would accept the registration of an oracle. Once an oracle's Ethereum address is part of the registered oracles, it becomes authorized to execute the functions in the COVID-19 Notification smart contract. A previously registered oracle can also become prohibited from accessing or executing the functions. This is also done through the 'Oracles SC'.

3) Oracles Contact Tracing Results: The registered oracles execute the contact tracing algorithm in response to the received red or yellow alert from the 'COVID-19 Notification SC'. Every alert sent by the 'COVID-19 Notification SC' possesses an ID to clearly label and identify it. The list produced by the oracles is assembled off-chain and its hash is computed. The list hash is then sent to the COVID-19 Notification SC. Each registered oracle needs to respond within the time allocated by the smart contract. The oracle has to send the list hash to the smart contract along with the request ID. Once the deadline is reached the timer oracle would then call the COVID-19 Notification SC to start comparing the received hashes and to declare the majority hash. The majority hash is the hash that matches at least $50 \%$ of the compared hashes. Figure 3 shows the communication details between the registered oracles and the COVID-19 Notification CS. It illustrates how the oracles execute the contact tracing algorithm and then send the hash to the smart contract for comparison and further computations.

4) Oracles Rewards and Penalty: The first oracle to have submitted a hash matching the majority hash is considered the winning oracle and is rewarded for its timely and accurate response. Oracles should be rewarded and penalized based on their behavior and output results [27]. This is done through digital assets, Ether as well as reputation. The reputation of oracles depends on how well they execute their tasks, their honesty, and their trustworthiness. Hence, each registered oracle is associated with a reputation that gets affected based on its performance. The winning oracle is rewarded with Ether to encourage other oracles to try and respond promptly.

\section{IMPLEMENTATION DETAILS}

This section presents the proposed algorithms along with their implementation and coding details. The solidity code is written and tested using the Remix IDE [28]. Further discussion is provided in the following subsections.

\section{A. Proof of Locations}

Algorithm 1 shows the details of the TriggerLocationAlert function available in the 'Location
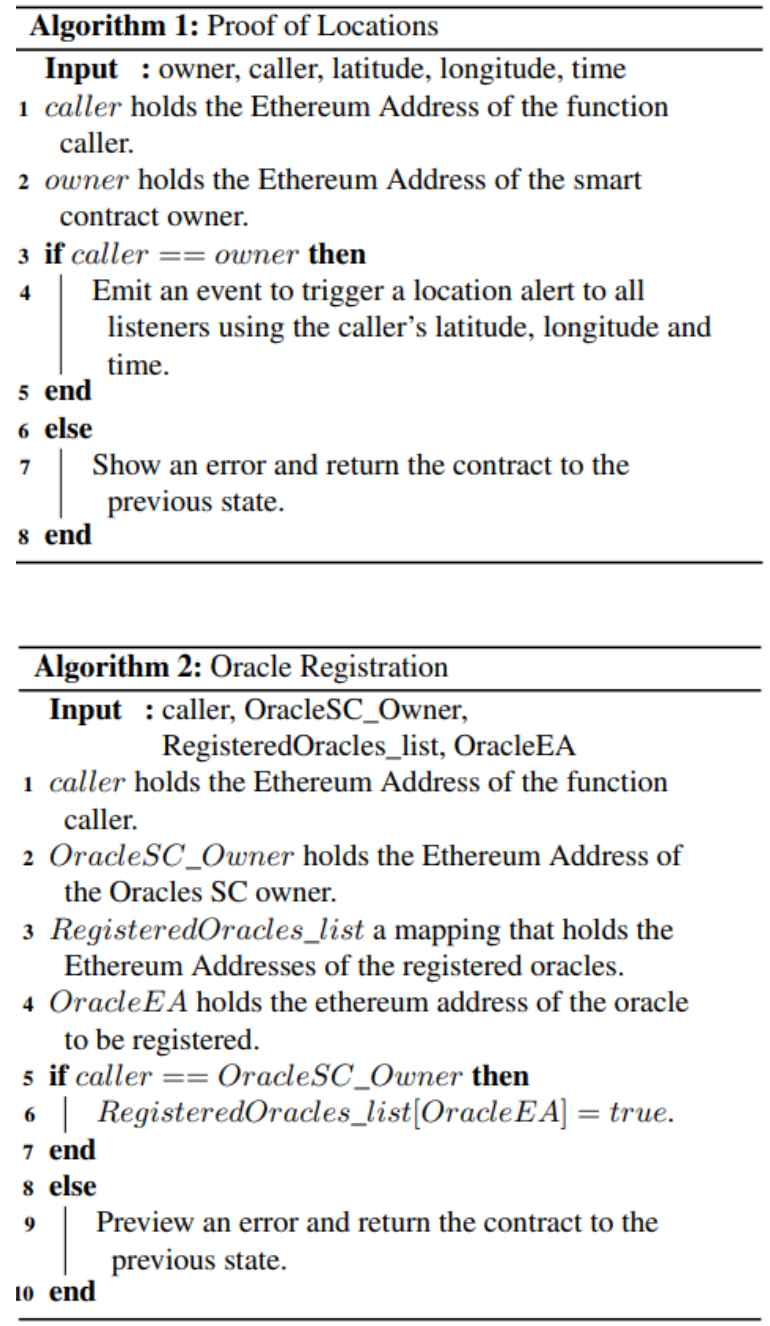

Tracking SC'. In this function, proof of locations of the mobile applications users is emitted by the smart contract based on the input received. The notification that is alerted contains the longitude, latitude, EA of the application user as well as the time.

\section{B. Oracle Registration}

The oracles that participate in the smart contracts must be registered. Algorithm 2 presents the registration details of the oracles in the 'Oracles SC'. Oracle registration is only possible through the smart contract owner. Hence, the algorithm checks the EA of the function caller. Then a mapping is used to complete the registration where the EA of the oracle is mapped with the boolean 'true'.

\section{Oracle Revoking}

If an oracle can no longer participate with the smart contracts, then it is revoked by the Oracle SC owner. Algorithm 3 shows the details of revoking the previously registered oracle. This is done by mapping the EA of the oracle to a boolean value of 'false'. 


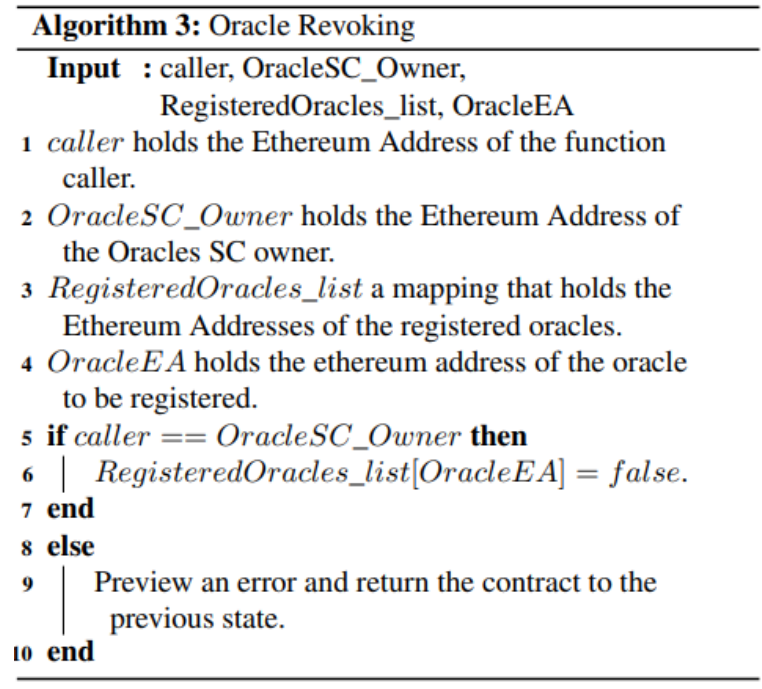

\section{Broadcast COVID-19 Test Results}

In the COVID-19 Notifications SC, the COVID-19 test results are sent by the testing centers to the smart contract. This is done through the SendCovid19TestResults function. Algorithm 4 explains the details followed in the function. The results show three types of events either a test taker is COVID19 positive, or a contact, or COVID-19 negative. Those results emit a red, yellow, or clear alert, respectively. Each result received by the testing center and sent on chain is given an ID. A red request ID and a yellow request ID are also maintained for tracing back easily when needed. The timestamp on chain as well as the request ID are stored every time a new request is created as can be seen in algorithm 4 . This algorithm can only be executed by the smart contract owner who is the testing center representative on chain. Therefore, a modifier is used to restrict access to the function accordingly.

\section{E. Return the Contact Tracing List Hash}

Once an event is emitted for a red or a yellow alert, the registered oracles' role commences. Each of the registered oracles should execute the contact tracing list algorithm to find out the EAs of all the contacted people who could be infected by the COVID-19 virus. Those EAs could be numerous. Consequently, a hash is sent by the registered oracles using algorithm 5. Each registered oracle calls the function using the request ID, request timestamp, and the list hash. The function as can be seen in the algorithm checks the EA of the oracle to ensure the oracle is registered. Furthermore, it verifies that the ID and timestamp match the ID and timestamp of the request ID. In addition to those two aforementioned restrictions, the oracle has to reply within the allocated time of 60 seconds, otherwise, the entry is refused. Moreover, this function will only accept entries for a particular request ID at a time. Hence, it processes the request IDs sequentially. It first accepts all the entries for the current request ID and then once that request's deadline approaches and the timer oracle ends the time for it, this function starts accepting entries for the next request ID. This is important to avoid making lists for each request ID at

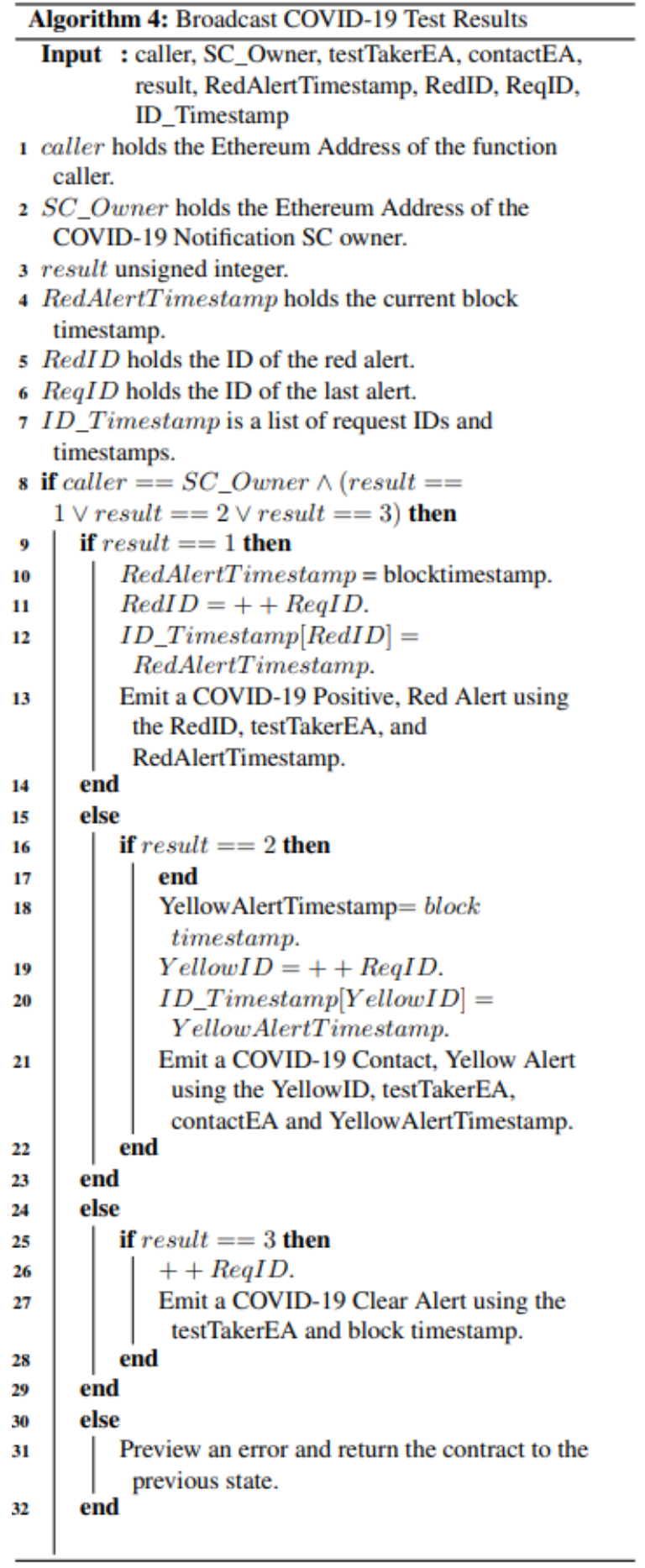

the same time which would cost a lot more compared to the current approach where only one list is reused every time a new request ID is processed. Five restrictions are important to ensure the proper execution of this function. Last but not least, the algorithm ensures that each oracle can only execute the function once. This is vital to ensure that it is fair for all the registered oracles. Also, this helps in mitigating the risk of having one oracle dominating and abusing their powers to deny others from casting their replies. Hence, to achieve this a unique value is stored every time an oracle submits its list 


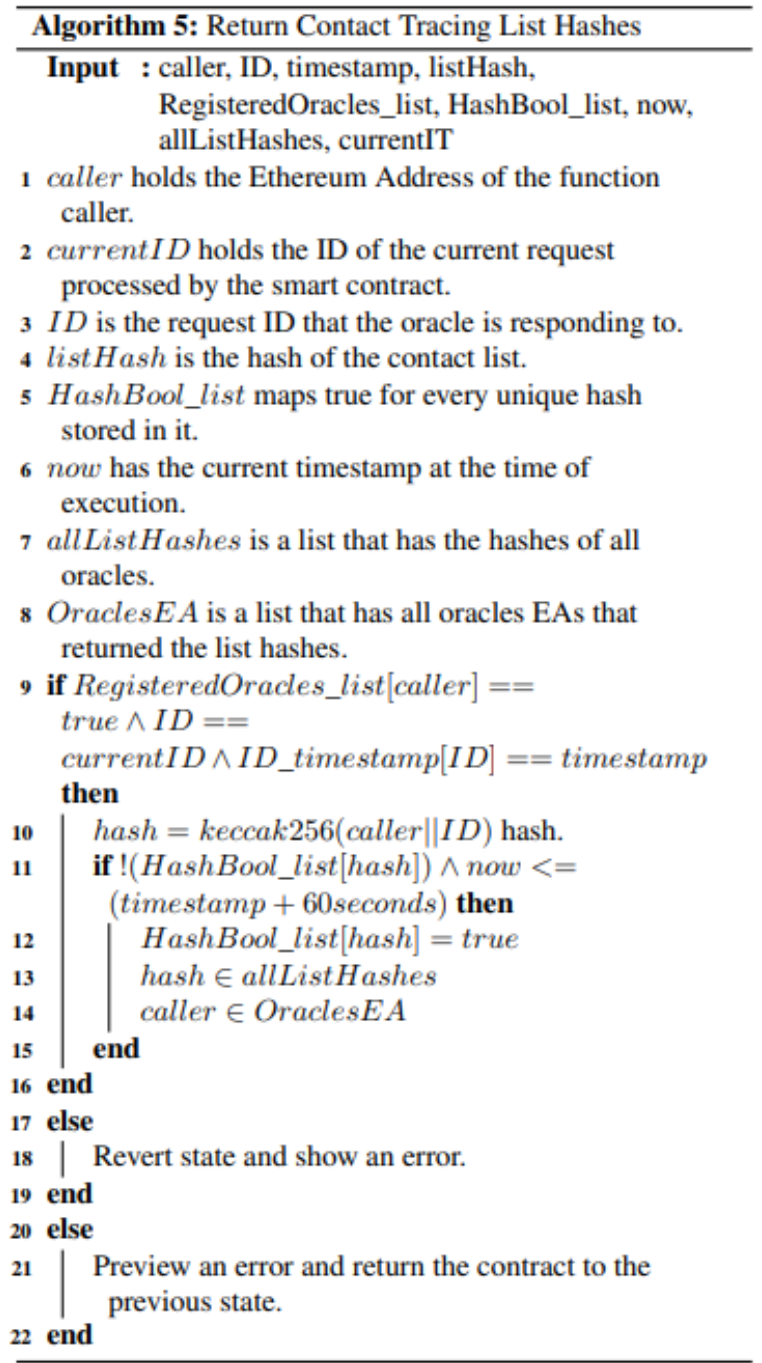

hash. This unique value is the keccak256 hash of the oracle's EA concatenated with the request ID.

We have used a combined index to ensure the value is unique for every entry submitted by an oracle. This combined index is then mapped to a boolean value. When an oracle returns a list for the first time, the combined index created is mapped to true. This value is checked every time an oracle returns a list. If the value returned is true, the check returns a false and the contract state is reversed. The keccak256 hash is used instead of other hashes as it is the least expensive in terms of gas cost. To be able to easily find the hash of the oracle's EA concatenated to the request ID using built-in solidity functions only, the keccak256 function of solidity accepts multiple values together if they are padded correctly. Hence, we ensured that no padding is done between both values when concatenated using the built-in function abi.encodePacked. This function concatenates both values in a byte array in the memory without changing their values. Consequently, in solidity we used the following line of code to hash and concatenate as needed keccak256(abi.encodePacked(msg.sender, ID)).

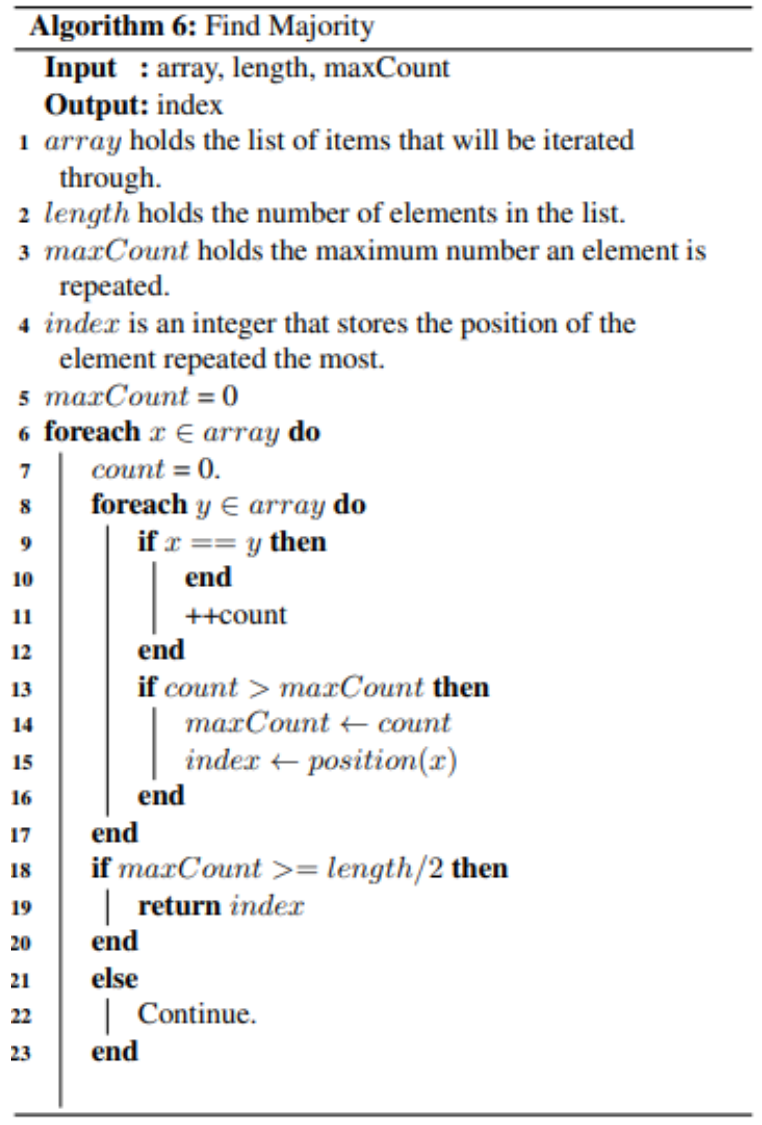

\section{F. Find Majority of the Contact List Hashes}

Algorithm 6 describes the details of finding the most common hash (majority) of all the received list hashes. The smart contract finds out the most repeated hash of all the hashes submitted by the registered oracles. A maximum count is stored in the algorithm and is swapped when a new maximum is found. The majority value is concluded when the maximum count is equal to or exceeds half of the number of hashes submitted. The algorithm returns the index of the majority value as an output.

\section{G. Choose the Winning Oracle}

In algorithm 7, the timer oracle executes a function when the allocated time for the oracles to submit their hashes is over. When the deadline for the oracles to submit their hashes is reached, algorithm 6 is called from within algorithm 7. The returned index from algorithm 6 is then used to locate the EA of the first oracle that replied with the chosen winning hash. A notification is emitted with the oracle's EA and the winning hash. Once the winning oracle is chosen, the request ID that can now be handled by the smart contract is incremented by 1. Furthermore, all the arrays used for storing and choosing the majority hash and winning oracle for the previous ID are deleted to be ready for processing the new request.

\section{H. Submit Contact Tracing List EAs}

The selected winning oracle will then submit the EAs of all the individuals in the contact tracing list. This is done one 

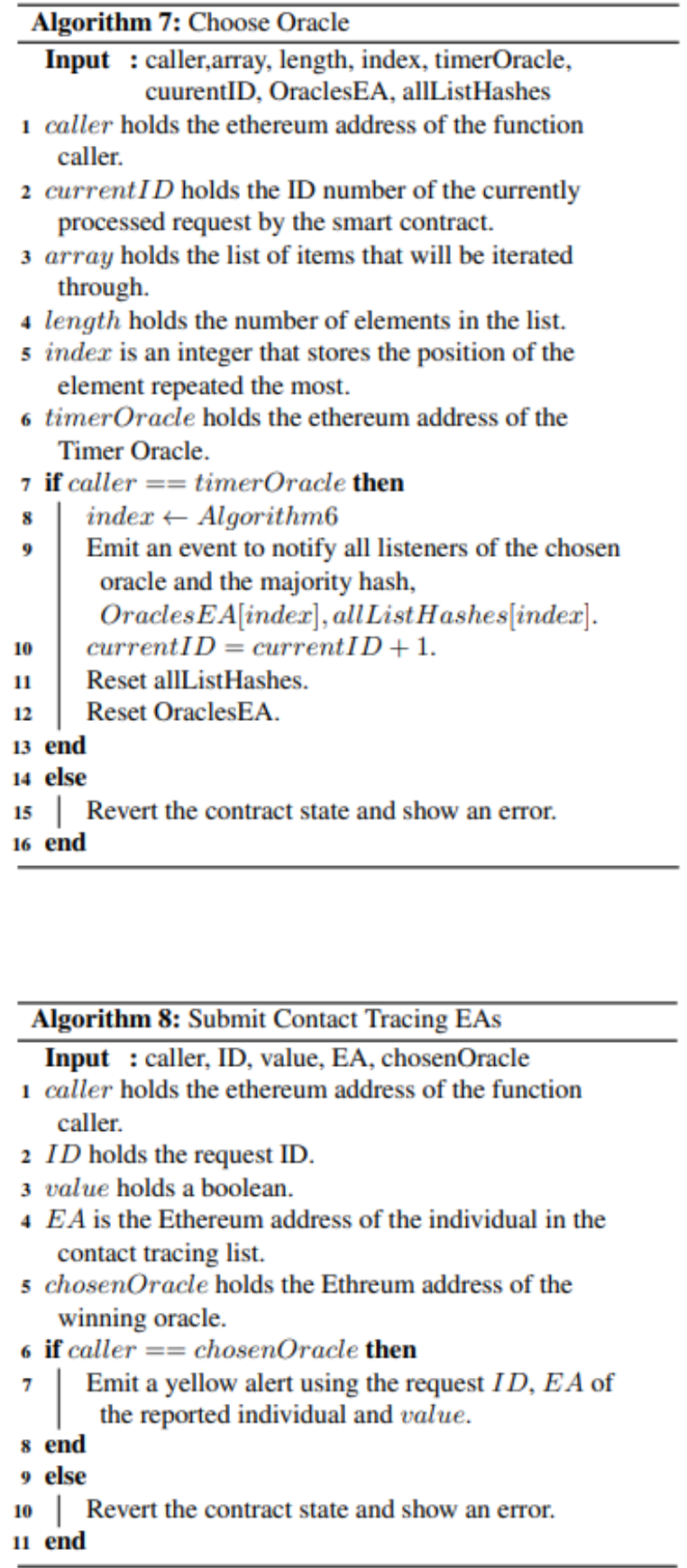

by one by executing the function EnterContactTracingList in the COVID-19 Notification SC. The function executes the algorithm described in algorithm 8 where the selected oracle can only be allowed to call the function. Furthermore, the EAs of the individuals are entered through the function as well as the request ID and a boolean value. The boolean is used to indicate if there are more function calls to be executed for the same request. For instance, when the chosen oracle reports the last EA, the boolean value reported would be false, unlike the previous calls where it was true. Whenever the function is executed, an event is emitted to issue a yellow alert introducing the individuals of the list using their associated EAs.

\section{Testing AND VALIDATION}

In this section, we rigorously tested the proposed smart contracts and presented their results. In our testing, the functions are tested for their functionality as well as the restrictions on them. Each function with a modifier to restrict the identity of the executor is tested with other EAs and the result is verified. Moreover, events and their logs are checked to ensure that they are as expected. Each smart contract has an owner. The owner could be a predefined EA in the smart contract or the EA of the entity that deploys the smart contract to the blockchain. The participating entities that interact with the smart contracts are the Location Tracking SC owner, the Oracles SC owner, the Notification SC owner, the registered algorithm tracing oracles, and the timer oracle. The functions are executed using the Remix [28] IDE and the results are shown in snapshots that showcase the function executed along with the results.

\section{A. Location Tracking SC: Location Alert Triggering}

A function called TriggerLocationAlert notifies by triggering an event every time when proof of location is sent to the blockchain. Figure 4 shows the latitude, longitude, user EA as well as the time successfully emitted as an event to all the participating entities as part of the immutable logs.

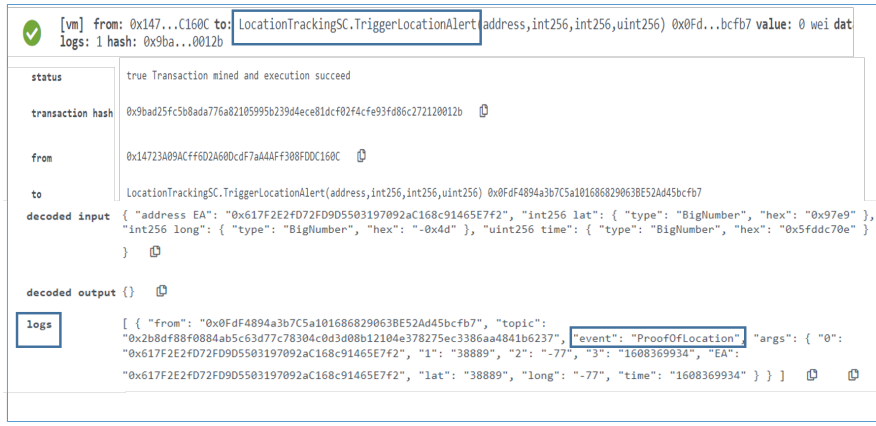

Figure 4: Logs showing a successful proof of location event

\section{B. Oracle SC: Oracle Registration}

Oracles need to be registered to execute functions call in the smart contract. Hence, the RegisterOracle function is used to register the oracles as can be seen in figure 5. The registration is performed using the oracle's EA. The EA is successfully added to the list of registered oracles mapping. Using this method, any oracle that tries to execute a function is first checked using an internal function in the Oracle SC to ensure that it is registered. The internal function returns a boolean which is the value mapped to the EA of the oracle at the time of the registration. 'True' is the boolean value mapped when registering the oracle. The registration can only be done by the owner of the Oracle SC. While testing, the Oracle SC owner

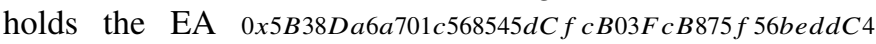
and the EA of the successfully registered oracle is $0 x d D 870 f A 1 b 7 C 4700 F 2 B D 7 f 44238821 C 26 f 7392148$ as can be seen in figure 5 . 


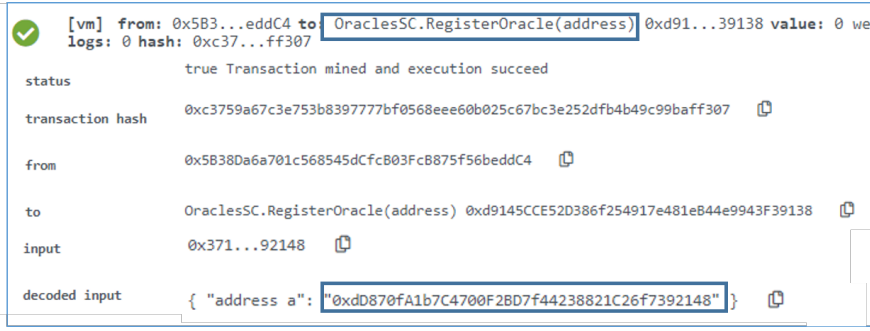

Figure 5: Logs of an oracle successfully registered in the Oracle SC

\section{Oracle SC: Oracle Revoking}

An oracle may no longer be trusted. Hence, a previously registered oracle with a boolean mapping of true is revoked by changing its mapped value to false. Function RevokeOracle in the OracleSC is used to do so. The function once executed successfully changes the mapping and revokes an oracle. Figure 6 shows a successful execution of the function where the SC owner revoked the oracle with the EA $0 x d D 870 f A 1 b 7 C 4700 F 2 B D 7 f 44238821 C 26 f 7392148$ from its authorities.

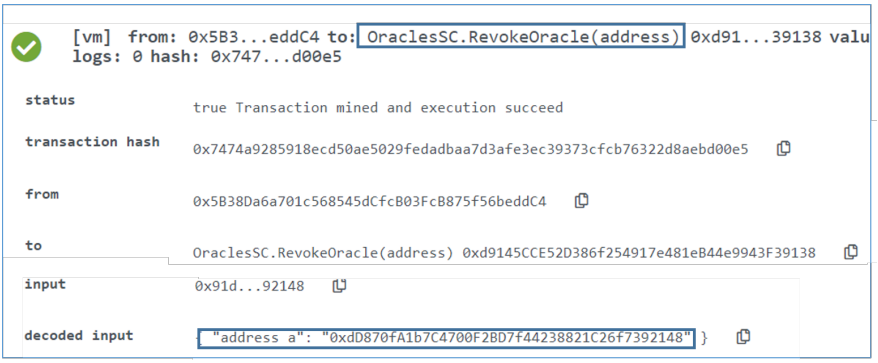

Figure 6: Logs of successfully revoking an oracle

\section{COVID-19 Notification SC: Broadcast COVID-19 Results}

The testing center communicates through the COVID-19 Notification smart contract all the COVID-19 test results. The alerts are then emitted by the smart contract based on the results as discussed earlier in the previous sections. Hence, the testing was successfully done as seen in figure 7. A successful red, yellow and green alert is presented based on the passed result in the input. The EA of the test taker is also part of the emitted event. Moreover, if a yellow alert is emitted, the EA of the test taker as well as the direct contact the disease was passed from are both parts of the emitted event. The event also documents the time and the type of alert. The result is also part of the alert. Therefore, in figure 7 the result is COVID19Positive since the alert emitted is a red alert. The results Contact or Clear are for a yellow and green alert respectively. Furthermore, a unique request ID is also emitted with every event as shown in figure 7 where the request ID for this event is 1 .

\section{E. COVID-19 notification SC: Return Contact Tracing List Hash}

The function ReturnHashes in the COVID-19 Notification $\mathrm{SC}$ is used by the registered oracles to return the contact

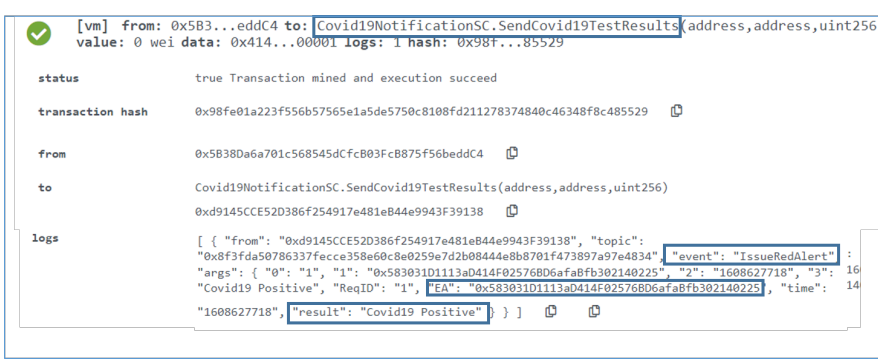

Figure 7: Logs of successfully emitting a red COVID-19 positive alert

list hash. Upon being alerted with a yellow or red alert, the registered oracles execute the contact tracing algorithm and return the list hash to the smart contract. The registered oracle uses the request ID, request timestamp and the list hash when executing the function. In figure 8 , the registered oracle 0xAb8483F64d9C6d1EcF9b849Ae677dD3315835cb2 entered the request ID which is 1 and the request timestamp 1608627718 as well as the list hash which is in bytes 32 format as seen here, ["0x64","0xEC","0x88","0xCA","0x00","0xB2","0x68",,0xE5","0xBA","0x1A","0x35",

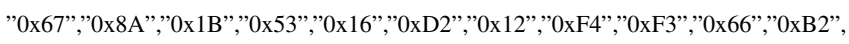

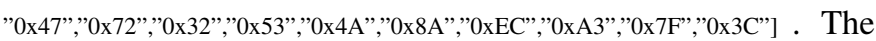
function is executed successfully and the hash is stored in the smart contract.

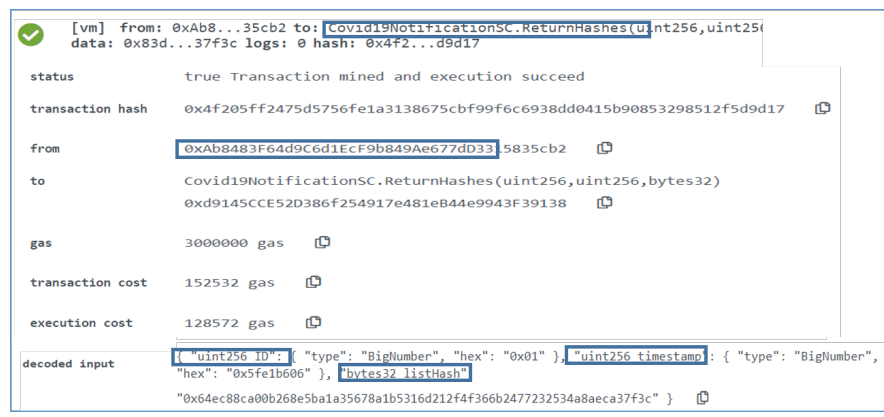

Figure 8: Logs showing a list hash successfully returned by a registered oracle

\section{F. COVID-19 Notification SC: Choosing the Winning Oracle}

All registered oracles try to submit on time the hash list after executing the contact tracing algorithm. The timer oracle, holder of the EA 0xdD870fA1b7C4700F2BD7f44238821C26f7392148 executes the ChooseOracle function in the COVID-19 Notification SC when the time for the oracles to return the hashes is finished. The function checks the majority hash which is the most repeated hash and then returns the first registered oracle that has returned that hash. The function is executed successfully by the timer oracle. The chosen list hash and the oracle that submitted the hash are announced in an event for all the participating entities as can be seen in figure 9 . The successful execution and testing of the ChooseOracle function also indicate the successful execution of the internal function findMajority function which is needed to find the majority hash that matches at least $50 \%$ of the submitted hashes in the list. 


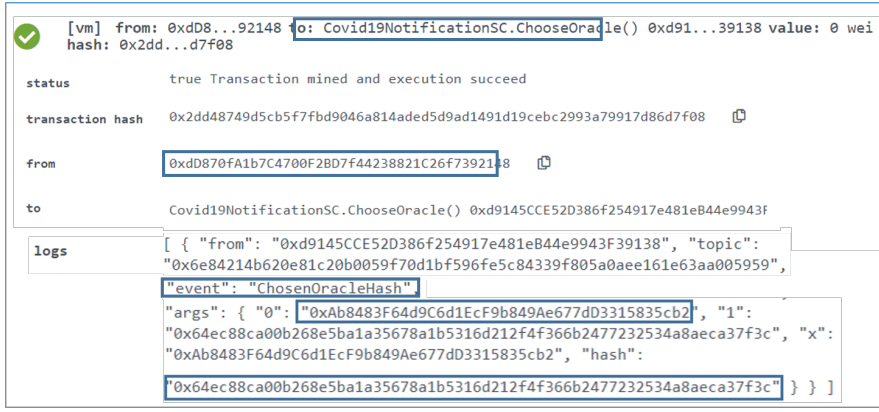

Figure 9: Logs showing a successful announcement of the chosen list hash and the winning registered oracle

\section{G. COVID-19 Notification SC: Submit Contact Tracing List EAs}

The winning oracle then needs to submit the EAs of the list to the smart contract. This is done through the EnterContactTracingList function. The function can only be executed by the winning oracle, otherwise, it would show an error and revert the contract state. The function takes the request ID, an EA, and a boolean which is only set to false to indicate the last item in the list. The EA submitted by the winning oracle is 0x0A098Eda01Ce92ff4A4CCb7A4fFFb5A43EBC70DC as can be seen in figure 10. This function emits an event that holds the details of the EA as well a contact tracing list yellow alert which can be used by the testing center for further action.

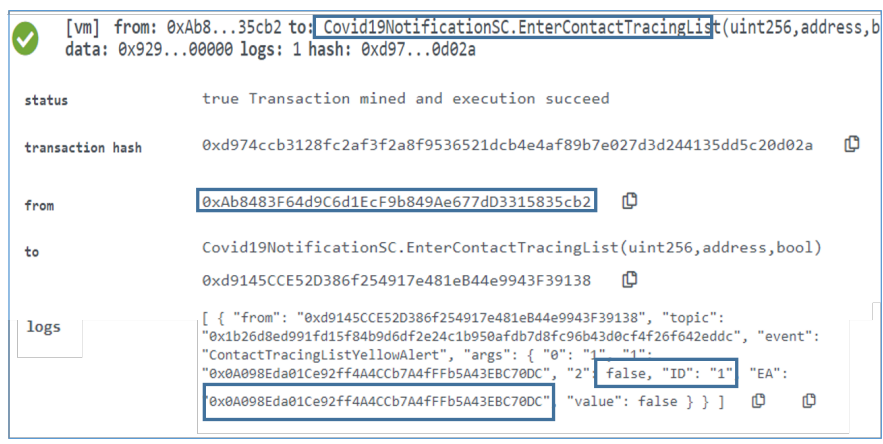

Figure 10: Logs indicating a successful entry of an EA in the contact tracing list

\section{Discussion}

In this section, we evaluate our solution using four parameters; namely, security, cost, privacy, and generalization aspects to measure its security and privacy strengths and verify its affordability, feasibility, scope, and practicality.

\section{A. Security Analysis}

Blockchain provides several intrinsic security features that are leveraged in our solution. Trusted and secure solutions can be built using its immutable and tamper-proof ledger. It eliminates exploits and vulnerabilities by incorporating authorization, availability, non-repudiation, accountability, integrity, and transparency. Each feature aforementioned is described below in detail with respect to our implemented solution.
Authorization is important to only allow designated entities to execute certain functions in the implementation. In our solution, every function in the smart contract can only be executed by a certain authorized entity. This is achieved through the usage of modifiers which help in checking the EA of the entity trying to execute the call and comparing it with the EA of the desired authorized entity. If the EA is not matching, then an error is shown and the smart contract state is revoked to the previous state.

Availability ensures a solution is robust, reliable, and trusted. A blockchain network is always available whenever needed. Any transaction can be executed at any time securely through the function calls in the smart contracts. Blockchain is also a decentralized and distributed ledger where each node has a local copy of all the transactions. Hence, the network is not prone to being a single point of failure or hacking, unlike centralized systems.

Moreover, Non-repudiation is an important and desirable feature that ensures no entity can deny its actions. Every transaction that is executed is part of the immutable logs and is digitally signed using the private key of the executor. Hence, no transaction on the chain is stored without details of the caller such as the Etherum address and the smart contract address. Furthermore, the digital signature clears any doubts related to the EA that executed the call as it implements accountability.

Furthermore, integrity is maintained through the tamperproof logs and immutable information available on-chain. Any data stored on-chain cannot be altered, added to, or deleted. All the transactions that are created are stored and preserved. They can be used for history tracking and tracing as the logs are well-maintained and resilient.

On the other hand, another desirable feature of contact tracing solutions is privacy. Although Ethereum is a public blockchain network, several other permissioned blockchain networks that depend on channels, groups, and Membership Service Providers (MSP) can enable private communication between groups and entities. In the context of contact tracing, it is important to ensure that the privacy of the users using the contact tracing application is not imperiled. Consequently, our design associates the EAs of the users on-chain to their biometric data when registering at testing centers [21]. Furthermore, our solution uses digital medical passports [21] and gives the freedom to the user to allow access to their data and information. Without authorization from the data owner, the information cannot be accessed or used [21]. Hence, the data stored from the contact tracing cannot be used for purposes that are not transparent to the users. Transparency is easily achieved by using the decentralized blockchain ledger. All the transactions on-chain are accessible to all participants. The information is not used for purposes that are not known to the users beforehand.

\section{B. Cost Analysis}

Any transaction executed on the ledger costs a certain fee. This fee is determined based on the current gas price (Gwei). Gwei is the price per unit of gas. Gas is used to determine 
how much is a transaction cost. As part of the logs, every executed transaction shows the transaction cost as well as the execution cost. The execution cost is a part of the transaction cost.

Gas prices keep fluctuating based on network congestion. Miners give high priority to transactions with higher gas prices. The more gas paid per transaction, the faster the miners would want to process it. Miners can decline a transaction if the gas price for it is too low and does not meet their set minimum threshold. In our cost analysis, we are using the gas prices found on the ETH Gas Station [29] on December 25, 2020. The prices for the fastest, fast, average, and cheap are $90,84,67$, and 50 Gwei, respectively. In the I we have used the cheap gas price of 50 Gwei in order to calculate the cost. Moreover, we have used a price of 300 USD for each Ether.

Table I shows the transaction cost as well as the execution cost in Gwei for algorithm 7 in the Notification SC. The table shows how as the number of elements increase in the array, the cost increases. Here, the number of elements represent the number of registered oracles that have responded before the deadline of a request. The cost is not negligible as can be seen from the table. However, the cost varies depending on the order of the array elements as well as their values. For instance, if $50 \%$ of the oracles responded with the same hash, the cost would be less compared to other scenarios where the oracles responding with different hashes are the first elements in the array. The cost in the table is expected since this algorithm has loops to choose the majority hash and element. Furthermore, the arrays are reset after every request. Once, the winning oracle is chosen the arrays are reset to use the same arrays for the next request ID.

A way of reducing the cost is possible by ensuring the registered oracles are honest and reliable. For instance, they need to be able to track that the current request ID the smart contract is taking hashes for is ID 1 for example. Therefore, they should all only reply back with the list hash for request ID 1 and should wait till the deadline is reached and the contract announces the new current ID is 2 for them to submit again. If this is ensured then there is no need to reset the array and the array can be looped from only the new elements added for ID 2 respectively. However, to ensure this is the case, the reputation of the oracles should be high and they should be trustworthy. Furthermore, watchdogs can also be used by other trusted and reputable registered oracles to ensure that all registered oracles are executing their tasks efficiently and honestly. This could greatly reduce the cost to only $\$ 0.8964$ for 10 elements in contract to 2.3817 as suggested by the table.

\section{Generalization}

Our solution is used to tackle an important and current problem which the world is facing due to the COVID-19 pandemic. However, although contact tracing is highly useful for COVID-19 to mitigate its aftermath effects, contact tracing can be used for any other contagious disease.

The presented blockchain-based solution can easily adapt to any other requirements of any contagious disease or application that requires contact tracing. Hence, this work is presented to help eradicate issues related to trust in contact tracing. Using blockchain and its intrinsic features as well as security characteristics make it ideal to establish trust, reliability, and a feasible solution. With the world now trying to lead a normal life in 2021, opening immigration as well as easing travel restrictions, contact tracing can greatly benefit to swiftly act if any case is determined as positive.

\section{Privacy Analysis}

Contact tracing applications involve several parties and require the cooperation of many entities to be successful. It has shown promising results to prevent the spreading of the infectious COVID-19 [2]. For the users to consider using contact tracing applications willingly, they need to be offered transparency, privacy, and accountability. It is the right of users to be offered privacy for their shared data and information while using the contact tracing application. Also, they have the right to choose and decide how much their data can be disclosed. This is implemented to ensure that the information of users is not prone to hacking or abuse.

Our solution uses Self-Sovereign Identity (SSI) to ensure that each user owns their identity data and they do not rely on a management system. Blockchain-based identity systems manifest on digital identities to eradicate relying on centralized servers and third parties. It is a user-controlled data management system [30]. On the other hand, Christopher Allen identifies SSI as a way that makes users the administrator of their identities that may be stored across several locations upon their consent. He also adds that the identity information of a user can be asserted or certified by other groups as well [31].

Furthermore, in our design, an EA is not associated onchain with any information that will reveal the true identity of the person. The information stored on-chain after registration as part of the medical digital passports only includes hashes. Hence, the true identity of a person is not revealed online and all transactions are made through only an electronic digital address. This kind of anonymity is similar to the anonymity used in Bitcoin where users are only known through electronic addresses [32]. Moreover, the proof of locations sent by the users on-chain are sent with a delay of 20 minutes to ensure the true location of a user is not known. This will preserve the privacy of users by maintaining their current location as confidential information.

\section{CONCLUSiON}

In this paper, we have proposed a decentralized blockchainbased contact tracing solution to mitigate the spread of COVID-19. We showcased how blockchain-based immutable logs can add trust, transparency, and accountability features into COVID-19 contact tracing applications. In our approach, we leveraged blockchain's built-in features to safeguard users' information when using contact tracing applications. Our solution preserves users' privacy by allowing them to choose when and to whom to share their information. We integrated the Ethereum blockchain with oracles to bridge the gap between the on-chain and off-chain data. We developed smart contracts, 
Table I: Gas cost in USD of algorithm 7 for multiple array elements

\begin{tabular}{llll}
\hline \hline $\begin{array}{l}\text { Number of } \\
\text { Array Elements }\end{array}$ & $\begin{array}{l}\text { Transaction } \\
\text { Gas }\end{array}$ & $\begin{array}{l}\text { Execution } \\
\text { Gas }\end{array}$ & $\begin{array}{l}\text { Cost } \\
\text { USD }\end{array}$ \\
\hline 1 & 58508 & 39890 & 0.59835 \\
2 & 50991 & 36132 & 0.764865 \\
3 & 63072 & 42172 & 0.94608 \\
4 & 79577 & 50425 & 1.193655 \\
5 & 92628 & 56950 & 1.38942 \\
6 & 101703 & 61488 & 1.525545 \\
7 & 114806 & 68039 & 1.72209 \\
8 & 129447 & 75360 & 1.941705 \\
9 & 143916 & 82594 & 2.15874 \\
10 & 158783 & 90028 & 2.381745 \\
\hline
\end{tabular}

proposed eight algorithms, and discussed their full implementation and testing details. We evaluated the proposed approach using cost and security parameters that show it is affordable, practical, and secure enough against well-known attacks. The proposed solution also ensures privacy and can be easily adapted into different types of contact tracing applications as per their needs and requirements with minimal modifications. Hence, leveraging our solution for contact tracing applications can assist in curbing the spread of COVID-19.

\section{ACKNOWLEDGEMENT}

This publication is based upon work supported by the Khalifa University of Science and Technology under Awards No. CIRA-2019-001 and RCII-2019-002, Center for Digital Supply Chain and Operations Management.

\section{REFERENCES}

[1] M. E. Kretzschmar, G. Rozhnova, M. C. Bootsma, M. van Boven, J. H van de Wijgert, and M. J. Bonten, "Impact of delays on effectiveness of contact tracing strategies for covid-19: a modelling study," The Lancet Public Health, vol. 5, no. 8, pp. e452-e459, 2020.

[2] I. Braithwaite, T. Callender, M. Bullock, and R. W. Aldridge, "Automated and partly automated contact tracing: a systematic review to inform the control of covid-19," The Lancet Digital Health, 2020.

[3] A. B. Dar, A. H. Lone, S. Zahoor, A. A. Khan, and R. Naaz, "Applicability of mobile contact tracing in fighting pandemic (covid-19): Issues, challenges and solutions," Computer Science Review, p. 100307, 2020.

[4] L. Reichert, S. Brack, and B. Scheuermann, "Privacy-preserving contact tracing of covid-19 patients." IACR Cryptol. ePrint Arch., vol. 2020, p. $375,2020$.

[5] "New creative commons paper addresses ethical hurdles to contact tracing adoption," [Accessed on: Jan 22, 2021]. [Online]. Available: https://beyondstandards.ieee.org/new-creative $\backslash$ - -commons-paper-addresses-ethical-hurdles-to-contact-tracing-adoption/

[6] D. Sim and K. Lim, "Coronavirus: why aren't singapore residents using the tracetogether contact-tracing app," South China Morning Post, vol. $18,2020$.

[7] Z. Zheng, S. Xie, H.-N. Dai, X. Chen, and H. Wang, "Blockchain challenges and opportunities: A survey," International Journal of Web and Grid Services, vol. 14, no. 4, pp. 352-375, 2018.

[8] K. M. Khan, J. Arshad, and M. M. Khan, "Simulation of transaction malleability attack for blockchain-based e-voting," Computers \& Electrical Engineering, vol. 83, p. 106583, 2020.

[9] H. Hasan, E. AlHadhrami, A. AlDhaheri, K. Salah, and R. Jayaraman, "Smart contract-based approach for efficient shipment management," Computers \& Industrial Engineering, vol. 136, pp. 149-159, 2019.

[10] A. Sharma, S. Bahl, A. K. Bagha, M. Javaid, D. K. Shukla, and A. Haleem, "Blockchain technology and its applications to combat covid-19 pandemic," Research on Biomedical Engineering, pp. 1-8, 2020.

[11] R. W. Ahmad, K. Salah, R. Jayaraman, I. Yaqoob, S. Ellahham, and M. Omar, "Blockchain and COVID-19 pandemic: Applications and challenges," 2020
[12] N. Ahmed, R. A. Michelin, W. Xue, S. Ruj, R. Malaney, S. S. Kanhere, A. Seneviratne, W. Hu, H. Janicke, and S. K. Jha, "A survey of covid-19 contact tracing apps," IEEE Access, vol. 8, pp. 134577-134601, 2020.

[13] A. Sharma, S. Bahl, A. K. Bagha, M. Javaid, D. K. Shukla, and A. Haleem, "Blockchain technology and its applications to combat covid-19 pandemic," Research on Biomedical Engineering, pp. 1-8, 2020.

[14] A. Kalla, T. Hewa, R. A. Mishra, M. Ylianttila, and M. Liyanage, "The role of blockchain to fight against covid-19," IEEE Engineering Management Review, vol. 48, no. 3, pp. 85-96, 2020.

[15] M. M. Arifeen, A. Al Mamun, M. S. Kaiser, and M. Mahmud, "Blockchain-enable contact tracing for preserving user privacy during covid-19 outbreak," 2020

[16] H. Xu, L. Zhang, O. Onireti, Y. Fang, W. B. Buchanan, and M. A. Imran, "Beeptrace: Blockchain-enabled privacy-preserving contact tracing for covid-19 pandemic and beyond," arXiv preprint arXiv:2005.10103, 2020.

[17] W. Lv, S. Wu, C. Jiang, Y. Cui, X. Qiu, and Y. Zhang, "Decentralized blockchain for privacy-preserving large-scale contact tracing," arXiv preprint arXiv:2007.00894, 2020.

[18] L. Garg, E. Chukwu, N. Nasser, C. Chakraborty, and G. Garg, "Anonymity preserving iot-based covid-19 and other infectious disease contact tracing model," IEEE Access, vol. 8, pp. 159 402-159414, 2020.

[19] J. Song, T. Gu, X. Feng, Y. Ge, and P. Mohapatra, "Blockchain meets covid-19: a framework for contact information sharing and risk notification system," arXiv preprint arXiv:2007.10529, 2020.

[20] E. Mbunge, "Integrating emerging technologies into covid-19 contact tracing: Opportunities, challenges and pitfalls," Diabetes \& Metabolic Syndrome: Clinical Research \& Reviews, vol. 14, no. 6, pp. 1631-1636, 2020.

[21] H. R. Hasan, K. Salah, R. Jayaraman, J. Arshad, I. Yaqoob, M. Omar, and S. Ellahham, "Blockchain-based solution for COVID-19 digital medical passports and immunity certificates," IEEE Access, vol. 8, pp. 222 093-222 108, 2020.

[22] "Gartner identifies five emerging trends that will drive technology innovation for the next decade," [Accessed on: Jan 25, 2021]. [Online]. Available: https://www.gartner.com/en/newsroom/ press-releases/2020-08-18-gartner-identifies-five-emerging-trends $\backslash$ -that-will-drive-technology-innovation-for-the-next-decade

[23] H. Al-Breiki, M. H. U. Rehman, K. Salah, and D. Svetinovic, "Trustworthy blockchain oracles: review, comparison, and open research challenges," IEEE Access, vol. 8, pp. 85 675-85 685, 2020.

[24] A. Egberts, "The oracle problem-an analysis of how blockchain oracles undermine the advantages of decentralized ledger systems," Available at SSRN 3382343, 2017.

[25] G. Caldarelli, C. Rossignoli, and A. Zardini, "Overcoming the blockchain oracle problem in the traceability of non-fungible products," Sustainability, vol. 12, no. 6, p. 2391, 2020.

[26] J. Adler, R. Berryhill, A. Veneris, Z. Poulos, N. Veira, and A. Kastania, "Astraea: A decentralized blockchain oracle," in International conference on internet of things (IThings) and IEEE green computing and communications (GreenCom) and IEEE cyber, physical and social computing (CPSCom) and IEEE smart data (SmartData). IEEE, 2018, pp. $1145-1152$

[27] A. A. Battah, M. M. Madine, H. Alzaabi, I. Yaqoob, K. Salah, and R. Jayaraman, "Blockchain-based multi-party authorization for accessing ipfs encrypted data," IEEE Access, vol. 8, pp. 196813-196825, 2020. 
[28] "Remix," [Accessed on: July 27, 2020]. [Online]. Available: https: //remix.ethereum.org/

[29] "Eth gas station," [Accessed on: November 11, 2018]. [Online]. Available: https://ethgasstation.info/

[30] M. S. Ferdous, F. Chowdhury, and M. O. Alassafi, "In search of self-sovereign identity leveraging blockchain technology," IEEE Access, vol. 7, pp. $103059-103079,2019$.

[31] Q. Stokkink and J. Pouwelse, "Deployment of a blockchain-based selfsovereign identity," in International conference on Internet of Things (iThings) and IEEE green computing and communications (GreenCom) and IEEE cyber, physical and social computing (CPSCOm) and IEEE smart data (SmartData). IEEE, 2018, pp. 1336-1342.

[32] F. Reid and M. Harrigan, "An analysis of anonymity in the bitcoin system," in Security and privacy in social networks. Springer, New York, NY, 2013, pp. 197-223. 